\title{
RESONANCE FOR QUASILINEAR HYPERBOLIC EQUATION ${ }^{1}$
}

\author{
BY TAI-PING LIU
}

In this note we announce results on a nonlinear conservation law with a moving source.

$$
\frac{\partial u}{\partial t}+\frac{\partial f(u)}{\partial x}=g(x-d t, u)
$$

The equation is intended to model fluid motions under external effects, either physical or geometrical, such as gas flows through a nozzle, MHD shock tube [1, 6, 9]. As such, we assume that the flux $f(u)$ is a smooth convex function of the density $u$, and the source term has the form

$$
g(\xi, u)=c(\xi) h(u), \quad \xi=x-d t,
$$

where $c(\xi)$ is a piecewise continuous function and $h(u)$ is a smooth positive function whose first derivative does not change signs. The external effect is assumed to be finite; for simplicity, we suppose also that $c(\xi)$ has compact support.

Our main interest is the behavior of nonlinear waves when the resonance occurs, that is, when the characteristic speed $f(u)$ is close to the speed $d$ of the source. The behavior of nonresonance waves for general systems of conservation laws with source terms has been studied in [6]. These waves are dynamically stable. As a first step to understand the resonance effects, we study the interaction of shock waves and rarefaction waves for the conservation law

$$
\frac{\partial u}{\partial t}+\frac{\partial f(u)}{\partial t}=0
$$

$[3,5]$ and the steady traveling waves with speed $d$; that is, solutions of

$$
\frac{d(f(u)-d u)}{d \xi}=c(\xi) h(u), \quad \xi=x-d t
$$

[6]. When a transonic shock wave $\left(u_{-}, u_{+}\right), f^{\prime}\left(u_{-}\right)>d>f^{\prime}\left(u_{+}\right)$, propagates through a steady traveling wave it accelerates (or decelerates) and therefore is unstable (or stable) if $c(\xi) h^{\prime}(u)$ is negative (or positive). More interestingly, as a

\footnotetext{
Received by the editors October 28, 1981. $35 \mathrm{~L} 67$.

1980 Mathematics Subject Classification. Primary 76H05, 35L65; Secondary 76E30,

Key words and phrases. Resonance, quasilinear hyperbolic, conservation laws, moving source, shock waves, nonlinear stability and instability.

1 Partially supported by NSF Grant and Naval Surface Weapons Center Independent Research Fund. 
rarefaction wave propagates through a steady traveling wave, the portion of the rarefaction wave for which resonance never occurs, that is, $f^{\prime}(u)$ is never equal to $d$, propagates through and eventually reemerges as a different rarefaction wave. On the other hand, a rarefaction wave reflects as a compression wave whenever the resonance is reached. Although the interaction of these elementary waves is quite complicated, the outcome of the interaction is a noninteracting wave pattern which can be analyzed easily.

The above study of the nonlinear stability and instability of elementary waves, and of the noninteracting wave patterns is then used to construct a random choice method using the elementary waves as building blocks. This scheme yields stable physical solutions. The convergence of the scheme is based upon an estimate of the total variation of the function $\varphi(u)$ defined by

$$
\varphi^{\prime}(u)=\frac{f^{\prime}(u)-d}{h(u)} .
$$

Since equation (3) is singular when the resonance occurs, the approximate solutions may have unbounded total variation. This makes it harder to prove the consistency of the scheme, which is in fact established by an elaborate wave tracing technique combined with the analysis of wave cancellations. Global bounded solutions are shown to exist when the initial data $u(x, 0)$ have bounded total variation T.V. and

$$
\begin{gathered}
\lim _{u \rightarrow \pm \infty} \varphi(u)>\max \{\varphi(u(-\infty, 0)), \varphi(u(+\infty, 0))\}+3 \text { T.V.*, } \\
\text { T.V.* } \equiv \int_{-\infty}^{\infty}|c(\xi)| d \xi+\text { tot. var. } \varphi(u(x, 0)) .
\end{gathered}
$$

Condition (4) does not require the inhomogeneous term $g(x-d t, u)$ to depend on $u$ sublinearly, as needed for standard existence theory for a quasilinear equation, $[2,4]$. Instead, it is essential that the flux function $f(u)$ be nonlinear. In other words, the present theory is a strictly nonlinear theory.

The idea of using the analytical studies of elementary waves to construct a stable numerical scheme can be generalized in principle to general systems of conservation laws with source terms. The analysis of nonlinear stability and instability of elementary waves, and noninteracting wave patterns has been carried out for gas flows through a duct of varying area $[7,8]$.

\section{REFERENCES}

1. R. Courant and K. O. Friedrichs, Supersonic flow and shock waves, Interscience, New York, 1948.

2. A. Douglis, Layering methods for nonlinear partial differential equations of first order, Ann. Inst. Fourier (Grenoble) 22 (1972), $141-227$.

3. J. Glimm, Solutions in the large for nonlinear hyperbolic systems of equations, Comm. Pure Appl. Math. 18 (1965), 695-715. 
4. S. Kruzhkov, First order quasilinear equations in several independent variables, Math. USSR-Sb. 10 (1970), 217-243.

5. P. Lax, Hyperbolic system of conservation laws. II, Comm. Pure Appl. Math. 10 (1957), 537-566.

6. T.-P. Liu, Quasilinear hyperbolic systems, Comm. Math. Phys. 68 (1979), 141-172.

7. - Transonic gas flows along a duct of varying area, Arch. Rational Mech.

Anal. (to appear).

8 , Nonlinear stability and instability of transonic gas flow through a nozzle, Comm. Math. Phys. (to appear).

9. G. B. Whitham, Linear and nonlinear waves, Wiley, New York, 1974.

DEPARTMENT OF MATHEMATICS, UNIVERSITY OF MARYLAND, COLLEGE PARK, MARYLAND 20742 
